\title{
El profesional en urgencias y emergencias: agresividad y burnout
}

\section{The professional in emergency care: aggressiveness and burnout}

\author{
C. Miret ${ }^{1}$, A. Martínez Larrea ${ }^{2}$
}

\section{RESUMEN}

La violencia contra profesionales y el síndrome de burnout, o desgaste profesional del personal sanitario, ha adquirido una enorme importancia en los últimos años, especialmente en los servicios de urgencias. Sólo una pequeña proporción de las agresiones al personal sanitario sale a la luz, pero hay un gran volumen de incidentes violentos sumergidos que no constan en ninguna parte. Se han creado protocolos y registros de agresiones en diversas comunidades para contar con datos precisos y poder tomar las decisiones más adecuadas y oportunas.

Las agresiones sufridas por los trabajadores se encuentran dentro del amplio abanico de riesgos que afectan a la seguridad y salud de los trabajadores sanitarios, ya de por sí sometidos a factores de estrés laboral elevados que conducen a altos niveles de desgaste profesional (burnout).

Por otra parte, los "profesionales quemados" incrementan, con sus actitudes en el trabajo, el riesgo de agresiones contra sí mismos y contra sus compañeros. Las autoridades sanitarias tienen entre sus prioridades no sólo ofrecer un servicio de calidad a todas las personas usuarias del sistema sanitario, sino también que todo profesional sanitario pueda trabajar en las mejores condiciones laborales y con unos niveles de satisfacción, motivación y seguridad adecuados.

Palabras clave. Agresividad. Síndrome de burnout. Servicios de urgencias. Violencia laboral.

\begin{abstract}
Violence against professionals and the syndrome of burnout, or the professional exhaustion of health personnel, has acquired enormous significance in recent years, especially in emergency care. Only a small proportion of the aggressions against the health personnel come to light, but there is a great volume of submerged violent incidents that are not recorded anywhere. Protocols and registers of aggressions have been created in different autonomous communities to make precise data available so that more suitable and opportune decisions can be taken. The aggressions suffered by the workers fall within a wide range of risks that affect the safety and health of health workers, who are already subjected to high stress that lead to high levels of professional exhaustion (burnout). On the other hand, "burnt-out professionals", with their attitudes at work, increase the risk of aggressions against themselves and against their colleagues. The priorities of the health authorities include not only offering a quality service to all the users of the health system, but also ensuring that every health professional can work in optimum working conditions, with suitable levels of satisfaction, motivation and safety.
\end{abstract}

Key words. Aggressiveness. Burnout síndrome. Emergency care. Violence at work.
1. Servicio de Urgencias. Hospital de Mataró. Consorci Sanitari del Maresme. Barcelona.

2. Unidad de Hospitalización Psiquiátrica. Hospital Virgen del Camino. Pamplona.

\author{
Correspondencia \\ Alfredo Martínez Larrea \\ Unidad de Hospitalización Psiquiátrica \\ Hospital Virgen del Camino \\ Irunlarrea, 4 \\ 31008 Pamplona \\ E-mail: amartinl@cfnavarra.es
}




\section{INTRODUCCIÓN}

La violencia, por el número de víctimas y la magnitud de las secuelas que produce, se ha convertido en un problema de salud pública. La violencia se puede dar en todos los contextos donde se desenvuelve el ser humano y uno de éstos es el ámbito labo$\mathrm{ral}^{1-3}$.

En el lugar de trabajo, la violencia ha adquirido una enorme relevancia en los últimos años tanto en los países industrializados como en los países en desarrollo. Abarca diversos contextos laborales y grupos profesionales, afecta a la dignidad de las personas y es una fuente de desigualdad, estigmatización y conflicto en el trabajo $^{4-6}$. La víctima se siente humillada, desmotivada, con pérdida de confianza y autoestima. Al mismo tiempo, la violencia en el lugar de trabajo aparece como una amenaza grave, y a veces letal, contra la eficiencia y el éxito de las organizaciones y es causa de perturbaciones inmediatas y a largo plazo $^{7-10}$. La empresa u organización afectada ve deteriorada su imagen corporativa, las relaciones interpersonales, la organización del trabajo, la productividad, el ambiente laboral y la atención a los clientes.

El término "violencia" circunscrito al lugar de trabajo, Chapell y Di Martino lo definen como: "cualquier incidente en el cual un empleado es abusado, amenazado o atacado por un miembro del público". No sólo la violencia física provoca grandes impactos en la salud del trabajador sino también la que se infringe a través de un comportamiento repetitivo que gradualmente daña la integridad psicológica del afectado y que puede llegar a tener repercusiones de mayor cuantía que la violencia física ${ }^{11}$.

El estrés laboral crónico constituye una agresión continuada a la salud de los profesionales sanitarios que afecta a todos los profesionales, especialmente en los servicios de urgencias hospitalarios y extrahospitalarios. Como respuesta a esta agresión surge el síndrome de desgaste profesional (SDP) o "burnout». Afecta a la salud física y mental así como a las relaciones sociales de los trabajadores que lo padecen. Se han detectado síntomas de cansancio físico y emocional, tensión $\mathrm{y}$ ansiedad, alteraciones en las relaciones laborales, disminución de la eficacia y eficiencia en el trabajo, incremento del absentismo laboral y deterioro general de la calidad asistencial. En ocasiones, da lugar a entidades psicopatológicas bien definidas, depresión, ansiedad y trastornos de adaptación.

Se han detectado factores de personalidad que favorecen o previenen la aparición del SDP. También se han detectado factores ambientales vinculados a la aparición de este síndrome: insatisfacción laboral, falta de reconocimiento social, falta de identificación con la organización, menor control sobre el trabajo, autopercepción de baja remuneración económica y el hecho de atender a personas que padecen enfermedades o procesos terminales.

En el presente artículo pretendemos revisar, describir y analizar la realidad de las agresiones profesionales y del SDP para poder abordarlos con mayor eficacia a nivel personal e institucional.

\section{VIOLENCIA EN LOS CENTROS SANITARIOS}

Es muy difícil disponer de información real sobre las agresiones a las personas que trabajan en los servicios sanitarios ya que sólo una pequeña proporción de estas conductas sale a la luz, pero hay un gran volumen de incidentes violentos sumergidos que no constan en ninguna parte.

Los sondeos disponibles indican que en torno al $60 \%$ del personal sanitario ha sido objeto alguna vez de alguna agresión física o verbal y que el $45 \%$ de las agresiones ocurren en urgencias. Conforme a datos de la Organización Mundial del Trabajo, el 25\% de las agresiones en el medio laboral se producen en el entorno sanitario ${ }^{12}$.

Dadas las dimensiones del problema y las dificultades para disponer de datos fiables, diversas instancias están trabajan- 
do para la implementación de registros de notificación de incidentes violentos ${ }^{13}$. Esto ha permitido describir la tipología y la prevalencia de la violencia en el sector sanitario, identificar los principales factores de riesgo de la misma y comparar los datos obtenidos con los de otras investigaciones de referencia.

La motivación de la violencia en los centros sanitarios difiere significativamente de la de otros lugares de trabajo, tales como almacenes y taxis, en los que la violencia se relaciona más a menudo con el robo. La violencia en centros sanitarios procede generalmente de pacientes $\mathrm{y}$, de vez en cuando, de familiares que se sienten frustrados, vulnerables y fuera de control.

\section{¿Quién es sujeto de riesgo?}

Aunque cualquier persona que trabaja en un centro sanitario puede ser víctima de la violencia, los trabajadores que realizan cuidados de salud, más frecuentemente mujeres, enfermeras, médicos y auxiliares, son quienes tienen más contacto directo con los pacientes y, por ello, un riesgo más alto. El resto de personal del centro no está exento de riesgos, incluyendo los miembros de seguridad del hospital.
Presentan mayores riesgos los que trabajan en unidades de emergencia, hospitales psiquiátricos, unidades de ancianos y centros de rehabilitación de adictos, entre otras. El porcentaje más importante de estos trabajadores en riesgo lo compone el equipo de enfermería ${ }^{14-20}$.

\section{¿Dónde pueden ocurrir las agresiones?}

Los actos violentos pueden ocurrir en cualquier zona pero sucede más frecuentemente en las salas de urgencias y especialmente en las de psiquiatría ${ }^{21}$.

\section{¿Cuáles son los efectos de la violencia?}

Pueden extenderse en intensidad e incluyen lesiones físicas graves, lesiones físicas leves, inhabilitación física temporal y permanente, trauma psicológico e incluso la muerte. La violencia puede también tener resultados negativos en la organización, tales como baja moral del trabajador, estrés laboral progresivo, mayor rotación de personal, merma de la confianza en la dirección y en los compañeros de trabajo y un entorno de trabajo hostil (Tabla 1) ${ }^{22}$.

Tabla 1. Efectos de la violencia contra el personal sanitario.

\begin{tabular}{ll}
\hline \multicolumn{1}{c}{ Efectos sobre la persona } & \multicolumn{1}{c}{ Efectos sobre la organización } \\
\hline - Disminución de la motivación, implicación y & - Incremento del absentismo laboral y pérdida de \\
rendimiento laboral & horas de trabajo \\
- Disminución de la autoestima & - Descenso de la calidad de la asistencia \\
- Despersonalización & - Deterioro del ambiente laboral \\
- Síntomas psicosomáticos & - Deterioro de la imagen institucional \\
- Alteraciones emocionales & - Incremento de los costes por medicina defensiva \\
- Pensamientos negativos sobre sí mismo, la profesión & - Insatisfacción de usuarios y profesionales \\
y la organización & - Deterioro de la relación entre profesionales y \\
- Afectación de los rendimientos cognitivos & usuarios \\
- Conductas de evitación en el trabajo & - Conflictos laborales \\
- Afectación a la realización profesional y personal & \\
- Afectación de las relaciones personales y sociales & \\
\hline
\end{tabular}


Tabla 2. Factores de riesgo que promueven la violencia.

- Trabajar directamente con las personas inestables, especialmente si están bajo la influencia de drogas o alcohol o tienen una historia de violencia o trastornos mentales

- Trabajar con personal insuficiente, especialmente durante las horas de comer y de visita

- Traslado de pacientes

- Esperas largas por el servicio

- Salas de espera abarrotadas e incómodas

- Trabajar solo

- Mal diseño ambiental

- Seguridad inadecuada

- Falta de capacitación del personal y de policías para impedir y controlar las crisis con los pacientes potencialmente volubles

- Abuso de drogas y alcohol

- Acceso a las armas de fuego

- Movimiento ilimitado del público

- Mala iluminación de pasillos, salas, estacionamientos y otras áreas

\section{¿Cuáles son los factores de riesgo para la violencia?}

Varios estudios indican que la violencia ocurre a menudo durante las épocas de alta actividad e interacción con los pacientes, por ejemplo en el horario de la comida y durante las horas de visita y en el traslado del paciente. Las agresiones pueden ocurrir cuando se niega el servicio, cuando se ingresa o se da el alta a un paciente contra su voluntad o cuando un trabajador del área médica procura fijar límites en la comida, bebida o uso del tabaco o del alcohol.

Los factores de riesgo para la violencia varían de un centro a otro dependiendo de la localización, del tamaño y del tipo de cuidados. Los factores de riesgo comunes para la violencia del centro se incluyen en la tabla $2^{13-22}$.

\section{ESTRATEGIAS GENERALES PARA PREVENIR LA VIOLENCIA}

Para prevenir la violencia en centros sanitarios, los directivos deben desarrollar un programa de salud y seguridad que incluya unos compromisos de gestión y de participación de los trabajadores en la identificación del peligro, formación en seguridad, salud y prevención del peligro, control e información. Se han descrito ciertos aspectos que podrían contribuir a la prevención de la violencia en los centros sanitarios $^{23-24}$. Entre ellos cabe destacar las medidas medioambiental: señales de emergencia, alarma y sistemas de supervisión; instalación de dispositivos de seguridad tales como detectores de metales para evitar que las personas armadas entren en la institución; instalación de otros dispositivos de seguridad como cámaras y buena iluminación en vestíbulos; guardias de seguridad por la noche en ciertas áreas de aparcamiento; diseño de las áreas de espera para acomodar y asistir a los visitantes y a los pacientes que puedan tener retraso en la asistencia; diseñar el área del "triaje» y otras áreas públicas para reducir al mínimo el riesgo de agresiones. Otras tipo de prevención incluye medidas administrativas: proporcionar medios de personal para impedir que se trabaje solo y para reducir al mínimo el tiempo de espera de los pacientes; restringir el movimiento del público en el centro mediante accesos controlados por tarjetas y desarrollar un sistema para alertar a personal de seguridad cuando se sospechan situaciones de violencia. Deben incluirse específicamente medidas formativas: proporcionar a todos los trabajadores entrenamiento para reconocer y manejar las agresiones, para la resolución de los conflictos y para mantener la conciencia del peligro existente. Y por último, es imprescindible adoptar medidas de tipo social que incluyan el desarrollo y actualización de un marco legal adecuado y sufi- 
ciente que actúe como factor preventivo y disuasorio, así como campañas de promoción de valores culturales que prevengan la violencia en general.

No obstante, los factores de riesgo de la violencia ocupacional son específicos para cada centro sanitario y sus circunstancias de trabajo, hecho que hace necesaria su evaluación y tratamiento individualizado en cada uno de los centros. En este sentido, estudios recientes confirman que, aunque la violencia ocupacional en el sector de la sanidad es universal, pueden variar sus características locales ${ }^{25-28}$.

Dado que la violencia puede ocurrir en el lugar de trabajo a pesar de medidas preventivas, los directivos deben estar preparados para ocuparse de las consecuencias de esta violencia, proporcionar un ambiente que promueva la comunicación abierta y desarrollar procedimientos escritos para divulgar y responder a la violencia. Los directivos deben ofrecer y proporcionar asesoramiento y atención siempre que amenacen o agredan a un trabajador ${ }^{29}$.

\section{ESTRÉS LABORAL Y SÍNDROME DE DESGASTE PROFESIONAL (BURNOUT)}

El estrés en un proceso dinámico complejo que viene definido por el desbordamiento de los recursos de la persona para hacer frente a las demandas del medio. Se considera que una persona está sometida a estrés cuando de forma real o distorsionada tiene la percepción de no ser capaz de hacer frente de forma efectiva a las demandas y exigencias del entorno.

El estrés laboral afecta a más del 25\% de trabajadores de todos los ámbitos y es una de las principales causas de absentismo laboral y pérdida de productividad. Estos costes se unen a los gastos de atención sanitaria que ocasiona y al sufrimiento y deterioro personal, del entorno familiar, laboral y social de los afectados ${ }^{30}$.

Un porcentaje significativo de los profesionales sanitarios refieren sentirse insatisfechos, desmotivados, desilusiona- dos y frustrados en su trabajo por numerosas causas de índole diversa y carácter crónico, relacionadas fundamentalmente con sobrecarga y estrés en el ámbito laboral.

El SDP o burnout fue definido por Freudenberguer en la década de los setenta y desarrollado por Maslach en los ochenta con la creación de su inventario que define las tres dimensiones clásicas del síndrome: cansancio emocional, despersonalización y falta de realización personal ${ }^{31}$. Dicho síndrome se origina como respuesta ante el estrés laboral e institucional continuado que sufren los profesionales, especialmente aquéllos que mantienen una relación constante y directa de ayuda con otras personas, sanitarios, educadores, etc. Este estado de sufrimiento mantenido, asociado al estrés laboral, termina afectando a la salud física de los profesionales. Se acumula cansancio de forma progresiva y se ven afectados los mecanismos básicos de recuperación, incluido el sueño. A nivel emocional, los profesionales refieren la sensación de estar dando más de lo que reciben y sienten que sus expectativas se ven repetidamente frustradas. Este cansancio emocional y físico es la primera manifestación del SDP.

Si no se reacciona a tiempo poniendo remedios adecuados, el profesional entra en una situación de alto riesgo. Puede presentar una distorsión significativa en su manera de percibir las amenazas y los agravios, reales o imaginarios, y reaccionar con estrategias disfuncionales para defenderse de estas percepciones. Se producen entonces cambios muy negativos de las actitudes profesionales. Progresivamente se desvaloriza la relación médico paciente y se sustituye por el distanciamiento emocional. Esto propicia que se responda con irritabilidad y cinismo ante las demandas de los pacientes y de la organización sanitaria. Se está entrando en la segunda dimensión del SDP: la despersonalización.

El trato despersonalizado a los pacientes y a sus familiares elimina la mayor fuente de satisfacción de las profesiones asociadas a las relaciones de ayuda, lo que refuerza el cansancio de los profesionales iniciando así 
un viaje sin retorno. Se agotan los recursos del profesional hasta incapacitarlo para el ejercicio de su profesión. Ésta puede ser la vía que nos conduzca a la tercera fase del SDP, la incompetencia personal o falta de realización personal con respuestas negativas hacia el trabajo y hacia sí mismo por parte de los profesionales "quemados" que han perdido total y en ocasiones definitivamente la motivación para el trabajo y que llegan a la falta total de implicación laboral.

Las consecuencias del desgaste profesional y de la violencia hacia los profesionales se solapan y potencian entre sí: alteraciones emocionales y conductuales, psicosomáticas y sociales, pérdida de eficacia laboral y alteraciones en la vida familiar, elevado nivel de absentismo laboral tanto por problemas de salud física como psíquica, con aparición de cuadros depresivos, ingesta de psicofármacos y consumo de alcohol y drogas.

\section{FACTORES DE RIESGO DEL SÍNDROME DE DESGASTE PROFESIONAL}

Los factores de riesgo del SDP son complejos (Tabla 3). Como en toda reacción adaptativa, existen factores personales, psicológicos y psicopatológicos que hacen a algunos profesionales más vulnerables que a otros. La autoestima, la afectividad y el optimismo previenen el SDP. Del mismo modo, la satisfacción profesional en la empresa ejerce como factor de protección del SDP. Pero realmente, de forma progresiva, factores de la organización y sociales negativos, externos a la persona están afectando cada vez más significativamente áreas muy sensibles de los servicios públicos esenciales como la sanidad, la educación y los cuerpos y fuerzas de seguridad, dando lugar a estrés elevado y desgaste progresivo del personal.

Tabla 3. Factores relacionados con el síndrome de desgaste profesional.

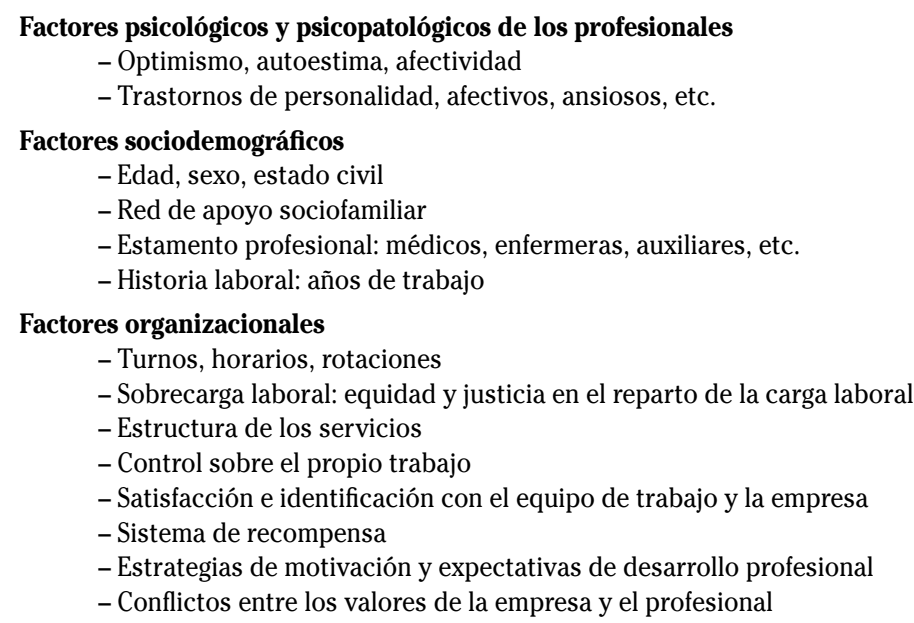

\section{Factores sociales}

- Valoración social de las profesiones sanitarias 
Los estudios sobre los efectos de la edad en el SDP han dado resultados no concluyentes. En profesiones con un elevado componente vocacional, la transición actual de las expectativas idealistas de la juventud y periodo de formación a la práctica clínica cotidiana puede frustrar con facilidad las expectativas de recompensas personales, profesionales y sociales. Así, tras los dos primeros años de carrera profesional se puede estar ya produciendo un fenómeno de sensibilización, puesto que los profesionales en esta época son especialmente vulnerables. Aunque inicialmente el SDP se describía en profesionales de más de 40 años, cada vez son mayores los indicadores de desgaste profesional entre los médicos residentes, especialmente en relación con las guardias en los servicios de urgencias ${ }^{32}$. Es importante tener en cuenta al evaluar estos datos el sesgo de supervivencia, puesto que los más afectados por el SDP son los que finalmente se ven abocados a abandonar el trabajo.

Se ven más afectados los profesionales que llevan más de 20 años de desarrollo profesional, con más de 10 años en el mismo centro de trabajo, especialmente si se trata de centros con gran número de profesionales del ámbito de la atención especializada y dedicados la mayor parte del tiempo de su jornada laboral a la atención directa de un número elevado de pacientes. Las mujeres y las personas que carecen de una pareja estable y del apoyo de una estructura sociofamiliar se han descrito como población de mayor riesgo, pero los resultados de los estudios son contradictorios. Los factores dependientes de la organización y el escaso apoyo social son causas de estrés laboral y pueden conducir al SDP. Las rotaciones laborales, los cambios de turno y de horario pueden incrementar el riesgo, especialmente en el personal de enfermería. La sobrecarga laboral, la estructura de los servicios, así como las expectativas de desarrollo profesional, el sistema de recompensas y el grado de control sobre el propio trabajo influyen. La presencia o ausencia de equidad y el conflicto entre los valores individuales y los de la empresa también influyen en el proceso de desgaste profesional.

De forma especialmente significativa, la valoración positiva del propio equipo de trabajo incrementa la realización personal, y la creencia en la utilidad del trabajo incrementa también los niveles de realización personal y protege frente a la despersonalización. Por el contrario, la creencia de que el trabajo no es valorado por los pacientes, familiares, compañeros y superiores incrementa los niveles de despersonalización. La eficacia y eficiencia del sistema sanitario se ha asentado sobre el compromiso fundamentalmente vocacional del personal sanitario. que precisa para su supervivencia sentirse integrado y partícipe en la organización como una parte fundamental de la misma y que su trabajo sea reconocido y valorado por la sociedad.

Los estudios demuestran que no todos los estamentos profesionales responden de la misma manera al estrés laboral y que el nivel de afectación en las distintas dimensiones del SDP puede variar en función de la profesión. Así, médicos y enfermeras están más afectados por el cansancio emocional, los médicos son los más afectados en la dimensión de despersonalización y los técnicos sanitarios y médicos los que obtienen resultados peores en la dimensión de realización personal ${ }^{33}$.

Los servicios de urgencias son ámbitos especialmente generadores de estrés, soportan una importante presión asistencial y altos niveles de responsabilidad, están sometidos a intensas exigencias por parte de los pacientes y familiares, excesivas horas de trabajo, falta de descanso, situaciones de riesgo y peligrosidad y, por ello, son lugares de especial riesgo para producir desgaste profesional.

\section{CONSIDERACIONES FINALES}

Se puede afirmar que la violencia contra los profesionales sanitarios y el síndrome de desgaste profesional se han convertido en un problema de salud pública, que afecta de forma creciente a los profesiona- 
les sanitarios, al propio sistema asistencial $\mathrm{y}$, por ende, a los pacientes, usuarios de los servicios sanitarios.

Ambos factores además están interrelacionados. Cuando con frecuencia atendemos a pacientes quejosos, en ocasiones con agresividad contenida o violencia manifiesta, nos refieren que el desencadenante de su estado ha sido la percepción de un trato deshumanizado e indigno, "sin ni siquiera mirarle a la cara". La despersonalización, fruto del desgaste profesional, incrementa el riesgo de agresiones contra el personal sanitario, y el ambiente hostil contra el personal sanitario incrementa el estrés y el desgaste profesional.

En determinados contextos y situaciones, tanto pacientes como profesionales pueden ser víctimas de deficiencias organizativas manifiestamente mejorables. Es necesario establecer planes de acción que incrementen la comunicación entre trabajadores y directivos para identificar áreas de riesgo y de mejora, planificar y realizar los cambios organizativos imprescindibles. Especial atención se ha de dar a las políticas incentivadoras, la equidad y la justicia en la distribución de cargas laborales y de recursos, el incremento de autonomía y responsabilidad de los profesionales y el reconocimiento social y empresarial del trabajo realizado.

$\mathrm{Al}$ mismo tiempo es necesario invertir en la formación específica de los profesionales y deben potenciarse los factores de protección, reforzando el optimismo y la autoestima y dotándolos de técnicas de autocontrol y gestión del estrés. Este esfuerzo se debe iniciar ya en los niveles de formación previos al acceso al mundo laboral. No podemos olvidar la necesidad de dotar al sistema de recursos para la detección y gestión de trabajadores con factores psicológicos y psicopatológicos que los hagan especialmente vulnerables.

Si los cuidadores se siente mal cuidados, están exhaustos, desmotivados y, sobre todo, no ven vías de solución a los problemas que les condujeron a esta situación, es lógico pensar que al final todo el sistema social se verá afectado.
Por pura inteligencia, la solución de estos problemas debería ser una prioridad social.

\section{BIBLIOGRAFÍA}

1. Artázcoz L, Moncada S. Organización del trabajo, satisfacción laboral y salud mental en trabajadores. Barcelona: Centro de Salud Laboral, Instituto Municipal de la Salud, 1997.

2. Avendaño C, Grau P. Riesgos para la salud de las enfermeras del sector público de Chile. Rev Enf 1997; 102: 15-26.

3. BARUdY J. Violencia agresiva y violencia ideológica en la fenomenología. En: Vilches $\mathrm{O}$ (Ed). Violencia en la cultura: riesgos y estrategias de intervención. Chile: Sociedad Chilena de Psicología Clínica 2000: 11-29.

4. Parent-Thirion A, Fernández E, Hurley J, VermeYLEN G. Fourth european working conditions survey. Dublin: European foundation for the improvement of living and working conditions, 2007.

5. WEILER A. Annual review of working conditions in the EU: 2005-2006. Dublin: European foundation for the improvement of living and working conditions, 2006.

6. Rumsey M, Foley E, Harrigan R, Dakin S. National overview of violence in the workplace. Australia: Royal College of Nursing, 2007.

7. Barudy J, Larraín S, Perrone R. Dinámica de la violencia. En: Vilches Álvarez O, editora. Violencia en la cultura: riesgos y estrategias de intervención. Chile: Sociedad Chilena de Psicología Clínica, 2000.

8. Burgos M, Paravic T. Percepción de violencia de los pacientes hospitalizados en los servicios de medicina y cirugía de un hospital público. Cienc Enferm 2003; 9: 29-42.

9. Burgos M, Paravic T. Violencia hospitalaria en pacientes. Cienc Enferm 2003; 9: 9-14.

10. Calvino ME. Género, salud mental y violencia. En: Gómez A (Ed). Los espejos de la desigualdad. Chile: Cuadernos Mujer Salud/6, 2001.

11. Chapell D, Di Martino V. Violence at work. Geneve: International Labour Office, 1998.

12. Defensor del Pueblo de Navarra. La violencia en el ámbito de la administración sanitaria. Informe especial al Parlamento de Navarra. Institución del Defensor del Pueblo de la Comunidad Foral de Navarra, 2008

13. Cervantes G, Cantera L, Blanch JM. Violencia hacia profesionales de la salud en su lugar de trabajo. Incidentes violentos notificados 
por internet en Catalunya entre 2005 y 2007 : resultados de un estudio piloto. Arch Prev Riesgos Laborales 2008; 11: 14-19.

14. Consejo Internacional de Enfermeras. Directrices para hacer frente a la violencia en el lugar de trabajo. Ginebra: CIE, 2007.

15. Consejo Internacional de Enfermeras. Enfermería y violencia. Boletín Enero-marzo de 1999.

16. OIT/ CIE/ OMS/ ISP. Directrices marco para afrontar la violencia laboral en el sector de la salud. Ginebra: Organización Internacional del Trabajo/ Consejo internacional de Enfermeras/ Organización Mundial de la Salud/ Internacional de Servicios Públicos. Programa conjunto sobre la violencia laboral en el sector de la salud, 2002.

17. Consejo Internacional de Enfermería. Nuevos estudios muestran que la violencia en el lugar de trabajo es una amenaza para los servicios de salud en todo el mundo. Comunicado de prensa. Extraído el 26 de septiembre de 2002.

18. Consejo Internacional de Enfermería. Directrices para hacer frente a la violencia en el lugar de trabajo. Ginebra, 1998.

19. Garza ME, León MA, Estada MT, Ramírez MG, LóPEZ ME, VELÁZquez U et al. Ansiedad laboral en personal de enfermería. Aten Primaria 1999; 23: 116-120.

20. Letelier P, Valenzuela S. Violencia: fenómeno relevante de estudio en campos clínicos intrahospitalarios de enfermería. Cienc Enferm 2002; 8: 21-26.

21. Gates D, Ross C, McQueen L. Violence against emergency department workers. J Emerg Med 2006; 31: 331-337.

22. Cantera LM, Cervantes G, Blanch JM. Violencia ocupacional: el caso de los profesionales sanitarios. Papeles del Psicólogo 2008, 291: 49-58.

23. MASIP JM. Prevención de riesgos laborales: estrategias, gestión y prevención de las adicciones en el mundo laboral. Madrid: Síntesis, 1999.
24. AMA. Violence in the medical workplace: prevention strategies. Chicago, IL: American Medical Association, 1995.

25. Felton JS. Violence prevention at the health caresite. Occup Med: State of the Art Rev 1997; 12: 701-715.

26. Goodman RA, Jenkins EL, Mercy JA. Workplacerelated homicide among health care workers in the United States, 1980 through 1990. JAMA 1994; 272: 1686-1688.

27. Lipsсомв J. Violence in the health care industry: greater recognition prompting occupational health and safety interventions. En: Charney W, editor. Essentials of modern hospital safety. Boca Raton, FL: Lewis Publishers 1994; 3: 29-104.

28. Department of health and human service, Centers for disease, control and prevention, National Institute for occupational Safety and Health. Occupational hazards in hospitals. DHHS (NIOSH) Publication No. 2002101.

29. SCHOPper D, LORMAND, JD, WAXWEILER R. Developing policies to prevent injuries and violence: guidelines for policy-makers and planner. Geneva: World Health Organization, 2006.

30. Bernal de Quirós-Aragón M, Labrador-Encinas FJ. Evaluación del estrés laboral y burnout en los servicios de urgencia extrahospitalaria. Int J Clin Health Psychol 2006; 7: 323-335

31. Atance Martínez JC. Aspectos epidemiológicos del síndrome de burnot en personal sanitario. Rev Esp Salud Pública 1997; 71: 293303.

32. Fernández Martínez O, Hidalgo Cabrera C, Martín Tapia A, Moreno Suárez S, García del Río GarCíA B. Burnout en médicos residentes que realizan guardias en un servicio de urgencias. Emergencias 2007; 19: 116-121.

33. Grau A, Suñer R, García MM. Desgaste profesional en el personal sanitario y su relación con los factores personales y ambientales. Gac Sanit 2005; 19: 463-470. 
